\title{
Management of children with interstitial lung diseases: the difficult issue of acute exacerbations
}

\author{
Annick Clement ${ }^{1}$, Jacques de Blic ${ }^{2}$, Ralph Epaud ${ }^{3}$, Laurie Galeron ${ }^{1}$, \\ Nadia Nathan ${ }^{1}$, Alice Hadchouel ${ }^{2}$, Angelo Barbato ${ }^{4}$, Deborah Snijders ${ }^{4}$, \\ Nural Kiper ${ }^{5}$, Steve Cunningham ${ }^{6}$, Matthias Griese ${ }^{7}$, Andrew Bush ${ }^{8}$ and \\ Nicolaus Schwerk ${ }^{9}$ on behalf of the chILD-EU collaboration
}

\begin{abstract}
Affiliations: 'Service de Pneumologie Pédiatrique, Hôpital Trousseau, Assistance Publique Hôpitaux de Paris (APHP), Sorbonne Universités, Université Pierre et Marie Curie (UPMC), Inserm U933, Paris, France. ${ }^{2}$ Service de Pneumologie Pédiatrique, Hôpital Universitaire Necker Enfants Malades, APHP, Université Paris Descartes, Paris, France. ${ }^{3}$ Service de Pédiatrie, Centre Hospitalier Intercommunal de Créteil, Inserm U955 (Eq.5), Université Paris-Est, Faculté de Médecine, Créteil, France. ${ }^{4}$ Dept of Women's and Children's Health, University of Padova, Padova, Italy. ${ }^{5}$ Dept of Pediatric Respiratory Medicine, Hacettepe University, Ankara, Turkey. ${ }^{6}$ Depat of Pediatrics, NHS Lothian and University of Edinburgh, Edinburgh, UK. ${ }^{7}$ Dept of Pediatric Pneumology, Dr von Hauner Children's Hospital, Ludwig-Maximilians-University, German Center for Lung Research, Munich, Germany. ${ }^{8}$ Dept of Pediatric Respiratory Medicine, Imperial College and Royal Brompton Hospital, London, UK. ${ }^{9}$ Clinic for Pediatric Allergology, Pneumology and Neonatology, Hannover Medical School, Hannover, Germany.
\end{abstract}

Correspondence: Annick Clement, Service de Pneumologie Pédiatrique, Hôpital Trousseau, APHP, UPMC, INSERM U933, 26 avenue du Docteur Arnold Netter, 75012 Paris, France. E-mail: annick.clement@aphp.fr

\section{0} @ERSpublications

The study provides the first proposal for acute exacerbation definition in paediatric interstitial lung diseases http://ow.ly/emPs305mpJ6

Interstitial lung disease (ILD) in children (chILD) is an umbrella term for a wide spectrum of rare diseases affecting the lung parenchyma, the causes of which often remain undetermined. These diffuse lung disorders are chronic, and often have high morbidity and mortality [1-3]. The epidemiology of the various forms of chILD is difficult to establish. Extrapolations from small studies have suggested an approximate incidence of $0.5-0.8$ cases per 100000 children $[4,5]$ However, this is certainly an underestimation due to the lack of standardised definitions, the inadequacy of organised reporting systems, and the variety of pathological conditions. In addition, clinical presentation is often nonspecific, contributing to a poor recognition of these disorders and confusion with other chronic pulmonary diseases. Insufficient disease-specific knowledge creates particular challenges for medical professionals, caregivers and chILD patients. Within the international community of clinicians and researchers involved in paediatric parenchymal lung diseases, the need for multicentre collaborations has resulted in the formation of networks of expertise to improve and harmonise approaches to diagnosis and management of the various forms of chILD. In this context, the present article reports expert opinions on the definition and diagnosis of acute exacerbations, which are major unpredictable deleterious episodes of acute worsening with significant morbidity that punctuate disease course.

Received: July 282016 | Accepted after revision: Oct 162016

Support statement: The project (Orphans Unite; chILD better together - European Management Platform for Childhood Interstitial Lung Disease) was funded by the FP7-305653-chILD-EU grant. A. Bush is an NIHR Senior Investigator, and additionally was supported by the NIHR Respiratory Disease Biomedical Research Unit at the Royal Brompton and Harefield NHS Foundation Trust and Imperial College London (London, UK). Funding information for this article has been deposited with Open Funder Registry.

Conflict of interest: None declared.

Copyright OERS 2016 


\section{Current challenges in chILD management}

The diagnosis of chILD is based on presenting history and clinical, radiological and functional abnormalities. Common features include dyspnoea, presence of diffuse infiltrates on chest imaging, and abnormal pulmonary function tests with evidence of a restrictive ventilatory defect (in older children) and/ or impaired gas exchange. It is now well recognised that the spectrum of disease expression and presentation is very heterogeneous. This is explained by the large variety of underlying causes, which are also influenced by the developmental processes of lung growth and maturation. As a consequence, no chILD classification schemes are entirely satisfactory at the present time. In this context of complexity and limited medical expertise, progress in improving chILD recognition and care remains slow [6].

Among the difficulties encountered in the various forms of chILD are the recognition and management of acute exacerbations. In all ILDs, acute exacerbations are probable critical events in the course of the disease and are associated with increased morbidity and mortality. The aetiology of acute exacerbations remains largely debated. The current understanding is that acute exacerbation represents an acceleration of the underlying pathological processes that may be precipitated by external triggers. Prospective studies are mandatory to elucidate causes. Therefore, we need a definition of acute exacerbations in order to ensure appropriate management and to define outcome parameters for clinical trials and other research studies.

A major difficulty is the lack of studies on acute exacerbations in chILD, so that working proposals have to be derived from other lung disease sources. In asthma, cystic fibrosis (CF), non-CF bronchiectasis and primary ciliary dyskinesia, acute exacerbations have sustained effects on disease progression and loss of lung function [7-12]. As such, they are increasingly being used as an endpoint in clinical trials. Therefore, the purpose of this article is to provide an update on the current understanding of acute exacerbations in children with various forms of ILD, and to propose acute exacerbation diagnostic criteria to improve patient management and to help define outcome parameters or clinical trials and other research studies. The work was conducted within the framework of the FP7 chILD-EU programme. It included a panel of experts in the field who provided recommendations based on opinion and knowledge. The project, which was launched in August 2015, was a combination of a critical literature review, online web-based surveys and face-to-face interactions, with the aim of obtaining a broad span of opinions and a comparison of experiences across clinicians from different countries [13]. We propose a minimal standard of acute exacerbation recognition that reflects current practice, but which has to be interpreted in the context of a limited amount of evidence. Finally, it is clear that further prospective studies are needed to develop more accurate indicators of acute exacerbations in chILD.

\section{Proposal for acute exacerbation definition}

The proposals for chILD acute exacerbation definition were first derived from a literature review focused on ILD. In adult patients with ILD and idiopathic pulmonary fibrosis (IPF), the first proposal was an acute clinically significant deterioration of unidentifiable cause in patients with underlying pulmonary fibrosis $[14,15]$. This clinical worsening could be related to an acceleration of the primary disease process and/or a consequence of occult complications such as infection or aspiration [16-21]. Even in adults, there are ongoing debates about AE definitions. Of importance, the prognosis of acute respiratory worsening in IPF was the same whether it was idiopathic or caused by identifiable triggers [22]. Therefore, acute exacerbations may be a deleterious event that accelerates parenchymal damage regardless of its cause. Consequently, the international working group on acute exacerbation in IPF recently defined acute exacerbations as an acute, clinically significant, respiratory deterioration characterised by evidence of new widespread alveolar abnormalities [23]. A categorisation of acute exacerbation based on the presence or absence of triggers was also proposed in IPF. The chILD expert panel rejected the distinction between idiopathic and secondary causes of acute exacerbation, largely because of the difficulty in determining causation [24]. In adults, most acute exacerbation studies are performed in IPF, which is a subgroup of ILD. However, all ILDs affect the lung parenchymal structure and homeostasis. Therefore, although extrapolation from IPF to other ILDs including chILD is undesirable, it is unavoidable given the lack of other good quality evidence, and we accepted this position. Consequently, the final chILD acute exacerbation definition was a significant worsening of the patient's respiratory condition that necessitates a change in regular management.

\section{Proposal for acute exacerbation diagnosis criteria}

Overview of acute exacerbation criteria selection process

The list of acute exacerbation diagnostic criteria that was first proposed by the expert panel was derived from studies performed in multiple lung diseases and patient groups. From the literature analysis, a list of possible criteria was established by identifying any items that could reflect a deterioration in the patient's condition. The severity of the worsening is a key parameter to take into account. However, no valid severity scores have been proposed so far. Consequently, the items were analysed and discussed 
independently, leading to a proposal of an initial list of 10 criteria. Next, these criteria were discussed in the context of the various clinical conditions, producing a final list of seven criteria (table 1). The duration of the deterioration before diagnosis of acute exacerbation was extensively debated. It was acknowledged that it depends on the severity of the changes. The final proposal was that an acute exacerbation would be expected to be diagnosed within 4 weeks of the changes first appearing. Lastly, most of the experts agreed that at least two criteria were needed to diagnose a chILD acute exacerbation.

\section{Clinical criteria and expert opinion}

Due to the rarity and heterogeneity of chILD, and the need for an all-age definition, the two initially proposed criteria that focused on nutrition could not be retained: unexplained feeding problems (after exclusion of other causes); and unexplained failure to thrive/weight loss (after exclusion of other causes). Indeed, there were concerns about the list of investigations that may be required in practice, the duration of nutritional difficulties to be considered, their severity, and their relevance in young or older children.

During the discussions, a consensus evolved about tachypnoea and dyspnoea. Measurement of respiratory rate is critical and, although the recommended method is to count the number of breaths in $1 \mathrm{~min}$, the techniques used in clinical practice are highly variable [25]. The expert panel acknowledged the absence of standard protocols across different hospital settings, as well as the difficulties in getting accurate results. They also discussed what a clinically significant alteration is in respiratory rate, considering that agreed levels are mandatory for clinical studies, especially for interventional trials. Finally, it was proposed that a $\geqslant 20 \%$ sustained increase in respiratory rate from baseline values was clinically significant $[11,13,26,27]$. This arbitrary proposal needs to be further evaluated in prospective studies. For the dyspnoea criterion, discussions were focused on evaluation of its severity. The sensation of difficult or uncomfortable breathing is a subjective experience perceived and reported by the patient and/or the family for young children. As there are no validated scoring instruments currently available in the paediatric population, no consensus could be reached.

\section{Functional criteria and expert opinion}

The criteria on oxygen desaturation and oxygen demand were initially proposed based on reports of other lung diseases. In most situations, however, blood oxygenation is evaluated by pulse oximetry which provides an estimation of arterial oxygen saturation $\left(\mathrm{SaO}_{2}\right) . \mathrm{SaO}_{2}$ results have difficulties in interpretation, as $\mathrm{SaO}_{2}$ can fluctuate spontaneously over time and can be influenced by many factors such as motion artifacts, poor perfusion, patient age and technical bias [28, 29]. At the present time, no recommendations on $\mathrm{SaO}_{2}$ thresholds for evaluating and monitoring children are available. In this context, the final proposal was to follow the decision of the referring physician to adjust oxygen therapy based on patient condition and the degree of desaturation. Consequently, the final wording was: onset and/or increase of oxygen demand to attain baseline saturation. The usefulness of adding functional tests to the list of criteria was

\section{TABLE 1 Acute exacerbation criteria in interstitial lung disease in children (chILD): initial and final list}

\section{Initial list of acute exacerbation criteria}

1. Increase in respiratory rate

2. Increase or development in/of dyspnoea

3. Newly developing abnormalities on chest imaging

4. Unexplained feeding problems lafter exclusion of other causes)

5. Unexplained failure to thrive/weight loss lafter exclusion of other causes]

6. Newly documented oxygen desaturation $(<93 \%)$ at rest and/or during exercise

7. Onset/increase of oxygen demand to attain the individual baseline saturation

8. Need for an additional level of ventilatory support

9. Decrease in lung function in children able to perform the tests

10. Reduced exercise tolerance in children able to perform the test ${ }^{\#}$

\section{Final list of acute exacerbation criteria}

1. Increase in respiratory rate $\geqslant 20 \%$ from baseline

2. Increase or development in/of dyspnoea

3. Newly developing or increased abnormalities on chest imaging

4. Onset/increase of/in oxygen demand to attain the individual baseline saturation (at rest and/or during exercise)

5. Need for an additional level of ventilatory support (in addition to oxygen)

6. Decrease in spirometry in children able to perform the tests $1 \geqslant 10 \%$ from baseline for vital capacity)

7. Reduced exercise tolerance in children able to perform the test ${ }^{\#}$

A panel of chILD experts was asked to provide a consensus proposal on acute exacerbation diagnosis criteria using a combination of critical literature review, online web-based surveys and face-to-face interactions. Participants rated the relevance and likelihood of use of the proposed criteria. The initial list included 10 acute exacerbation criteria. The final list retained seven acute exacerbation criteria. ${ }^{\#}$ : includes desaturation. 
debated. The conclusion was that they could provide important information. However, lung function assessment, especially in young children, may not be available or performed to international standards in all paediatric respiratory units [30]. The final decision was to include physiological tests in the list of acute exacerbation diagnostic criteria, restricted to those patients able to perform spirometry tests adequately. The level of alteration in respiratory rate was debated. Finally, it was proposed that a $\geqslant 10 \%$ decrease in vital capacity from baseline values was significant $[11,13,26,27]$.

\section{Radiological criterion and expert opinion}

Chest imaging was also extensively discussed. All agreed that radiological worsening as a sign of deterioration merited consideration. However, chILD is heterogeneous and radiological changes in acute exacerbation may be disparate. In addition, radiological changes may not be adequately documented on chest radiographs and may require serial high-resolution computed tomography. Ultimately, since in acute exacerbations in adult IPF radiological changes were considered critical [23], in keeping with the importance of harmonisation across the adults and children when possible, the expert decision was to keep this criterion in the final list.

\section{Conclusions and perspectives}

Development and validation of clinical tools to assess disease progression and treatment response are critical in chILD $[6,13,31]$. We propose a definition of acute exacerbations and select a set of diagnostic criteria, based on expert knowledge. This empirical approach only provides guidance in a rare and complex respiratory field. It is a first step that establishes a framework for the prospective studies needed to evaluate the current acute exacerbation definition, to validate or otherwise the criteria (especially considering the variability of some of the listed parameters), to document the prevalence of acute exacerbations in the various forms of chILD, to harmonise the search for specific triggers (e.g. infection and aspiration), and to determine management protocols for which the collaboration between the international chILD community and the patients and their families is mandatory.

\section{Acknowledgements}

We wish to thank all the participating teams who were involved in the online web-based surveys. The author contributions are as follows. M. Griese launched the project idea, and A. Clement and N. Schwerk designed the project. A. Clement and L. Galeron led the online web-based surveys. All authors participated in discussions for the development of the project. A. Clement and N. Schwerk drafted the manuscript. All authors reviewed the manuscript and agreed with the contents.

\section{References}

Clement A, Nathan N, Epaud R, et al. Interstitial lung diseases in children. Orphanet J Rare Dis 2010; 5: 22.

Kurland G, Deterding RR, Hagood JS, et al. An official American Thoracic Society clinical practice guideline: classification, evaluation, and management of childhood interstitial lung disease in infancy. Am J Respir Crit Care Med 2013; 188: 376-394.

3 Rice A, Tran-Dang MA, Bush A, et al. Diffuse lung disease in infancy and childhood: expanding the chILD classification. Histopathology 2013; 63: 743-755.

4 Hime NJ, Zurynski Y, Fitzgerald D, et al. Childhood interstitial lung disease: a systematic review. Pediatr Pulmonol 2015; 50: 1383-1392.

5 Griese M, Haug M, Brasch F, et al. Incidence and classification of pediatric diffuse parenchymal lung diseases in Germany. Orphanet J Rare Dis 2009; 4: 26.

6 Bush A, Anthony G, Barbato A, et al. Research in progress: put the orphanage out of business. Thorax 2013; 68: 971-973.

7 de Benedictis FM, Attanasi M. Asthma in childhood. Eur Respir Rev 2016; 25: 41-47.

8 Kapur N, Masters IB, Morris PS, et al. Defining pulmonary exacerbation in children with non-cystic fibrosis bronchiectasis. Pediatr Pulmonol 2012; 47: 68-75.

9 Sanders DB, Bittner RC, Rosenfeld M, et al. Failure to recover to baseline pulmonary function after cystic fibrosis pulmonary exacerbation. Am J Respir Crit Care Med 2010; 182: 627-632.

10 Sunther M, Bush A, Hogg C, et al. Recovery of baseline lung function after pulmonary exacerbation in children with primary ciliary dyskinesia. Pediatr Pulmonol 2016; [in press DOI: 10.1002/ppul.23479].

11 Bilton D, Canny G, Conway S, et al. Pulmonary exacerbation: towards a definition for use in clinical trials. Report from the EuroCareCF Working Group on outcome parameters in clinical trials. J Cyst Fibros 2011; 10: Suppl 2, S79-S81.

12 Oelsner EC, Loehr LR, Henderson AG, et al. Classifying chronic lower respiratory disease events in epidemiologic cohort studies. Ann Am Thorac Soc 2016; 13: 1057-1066.

13 Bush A, Cunningham S, de Blic J, et al. European protocols for the diagnosis and initial treatment of interstitial lung disease in children. Thorax 2015; 70: 1078-1084.

14 Raghu G, Rochwerg B, Zhang Y, et al. An Official ATS/ERS/JRS/ALAT Clinical Practice Guideline: Treatment of Idiopathic Pulmonary Fibrosis. An Update of the 2011 Clinical Practice Guideline. Am J Respir Crit Care Med 2015; 192: e3-e19.

15 Ryerson CJ, Cottin V, Brown KK, et al. Acute exacerbation of idiopathic pulmonary fibrosis: shifting the paradigm. Eur Respir J 2015; 46: 512-520. 
16 Johannson K, Collard HR. Acute exacerbation of idiopathic pulmonary fibrosis: a proposal. Curr Respir Care Rep 2013; 2: 10.

17 Lee JS, Collard HR, Anstrom KJ, et al. Anti-acid treatment and disease progression in idiopathic pulmonary fibrosis: an analysis of data from three randomised controlled trials. Lancet Respir Med 2013; 1: 369-376.

18 Johannson KA, Vittinghoff $\mathrm{E}$, Lee $\mathrm{K}$, et al. Acute exacerbation of idiopathic pulmonary fibrosis associated with air pollution exposure. Eur Respir J 2014; 43: 1124-1131.

19 Raghu G, Amatto VC, Behr J, et al. Comorbidities in idiopathic pulmonary fibrosis patients: a systematic literature review. Eur Respir J 2015; 46: 1113-1130.

20 Wootton SC, Kim DS, Kondoh Y, et al. Viral infection in acute exacerbation of idiopathic pulmonary fibrosis. Am J Respir Crit Care Med 2011; 183: 1698-1702.

21 Han MK, Zhou Y, Murray S, et al. Lung microbiome and disease progression in idiopathic pulmonary fibrosis: an analysis of the COMET study. Lancet Respir Med 2014; 2: 548-556.

22 Collard HR, Yow E, Richeldi L, et al. Suspected acute exacerbation of idiopathic pulmonary fibrosis as an outcome measure in clinical trials. Respir Res 2013; 14: 73.

23 Collard HR, Ryerson CJ, Corte TJ, et al. Acute exacerbation of idiopathic pulmonary fibrosis: an International Working Group Report. Am J Respir Crit Care Med 2016; 194: 265-275.

24 Houin PR, Deterding RR, Young LR. Exacerbations in neuroendocrine cell hyperplasia of infancy are characterized by increased air trapping. Pediatr Pulmonol 2016; 51: E9-E12.

25 Karlen W, Gan H, Chiu M, et al. Improving the accuracy and efficiency of respiratory rate measurements in children using mobile devices. PLoS One 2014; 9: e99266.

26 Horsley AR, Davies JC, Gray RD, et al. Changes in physiological, functional and structural markers of cystic fibrosis lung disease with treatment of a pulmonary exacerbation. Thorax 2013; 68: 532-539.

27 Morgan WJ, Wagener JS, Yegin A, et al. Probability of treatment following acute decline in lung function in children with cystic fibrosis is related to baseline pulmonary function. J Pediatr 2013; 163: 1152-1157.

28 Enoch AJ, English M, Shepperd S. Does pulse oximeter use impact health outcomes? A systematic review. Arch Dis Child 2016; 101: 694-700.

29 Fouzas S, Priftis KN, Anthracopoulos MB. Pulse oximetry in pediatric practice. Pediatrics 2011; 128: 740-752.

30 Jenkins BA, Glenn LL. Variability of FEV1 and criterion for acute pulmonary exacerbation. Front Pediatr 2014; 2 : 114.

31 Clement A. Task force on chronic interstitial lung disease in immunocompetent children. Eur Respir J 2004; 24: 686-697. 